\title{
|||||||||||||||||||||||||||||||||||||||||||||||||||||||||||||||||||.
}

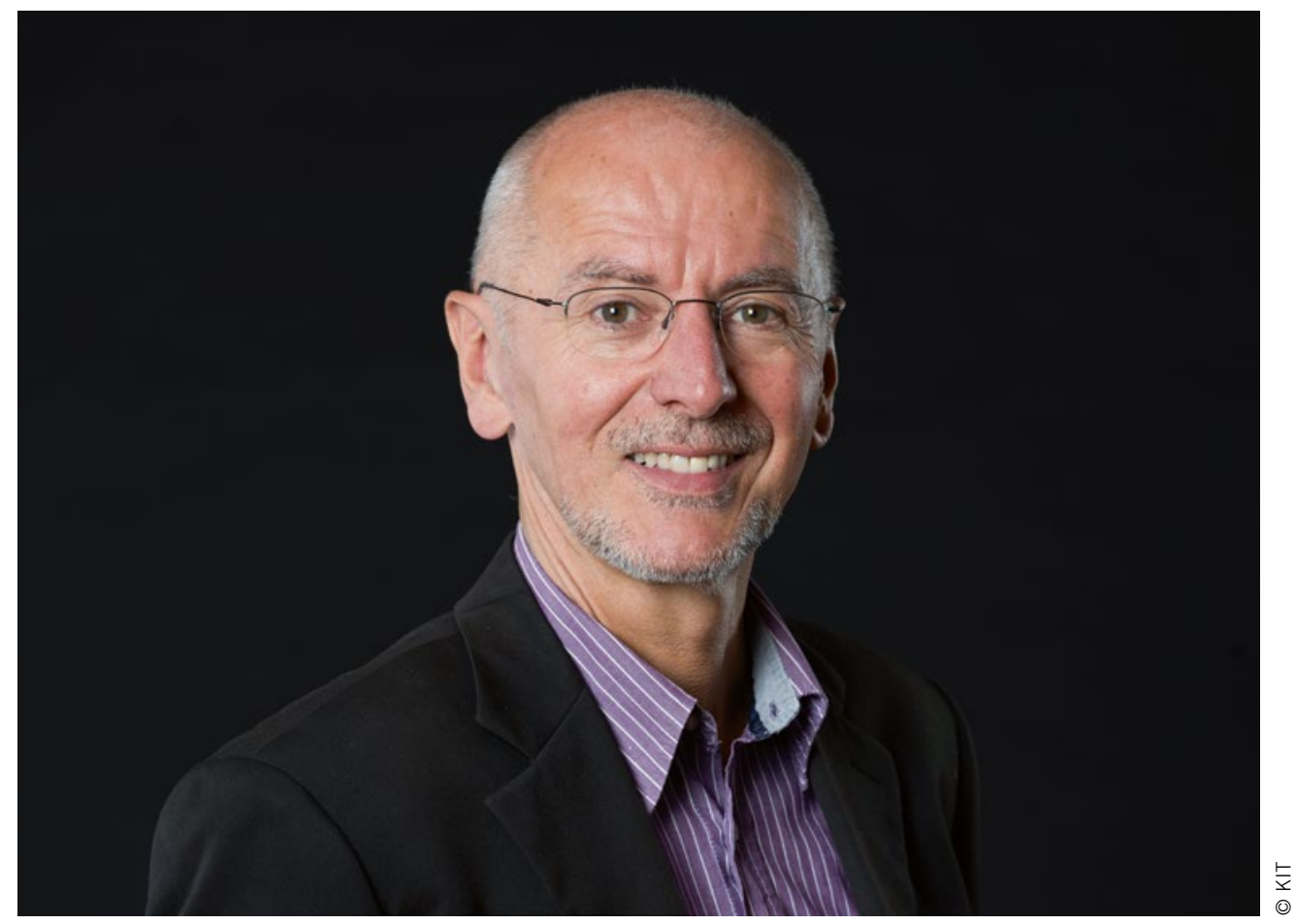

Prof. Dr. rer. nat

Armin Grunwald

Head of the Institute for

Technology Assessment

and Systems Analysis (ITAS)

at the Karlsruhe Institute of

Technology (KIT), Germany,

and Head of the Office of

Technology Assessment at

the German Bundestag (TAB)

\section{How Serious Are the Ethical Dilemmas?}

We are poised on the threshold of a new era. Soon we will have access to increasingly autonomous transport services and self-driving cars will start to appear on our roads. Advanced technology and self-learning algorithms are needed in order to achieve this, but we also have to overcome a number of ethical and legal problems.

The most familiar are extreme ethical situations. For example, a child runs out onto a highway in front of a car. There is traffic traveling in the opposite direction and the road is lined with trees. Every possible decision made by the car's computer would have negative consequences. A self-driving car may have to choose between running over two children or three older people. Or perhaps it has to decide whether to hit a homeless person with a $70 \%$ chance of being killed or a top performer of society with a $30 \%$ chance. How can we develop algorithms that provide the perfect ethical and legally defensible solutions in these cases?

The media often claim that the situation is hopeless and that autonomous vehicles should not be let loose on our streets, because they will give rise to insoluble questions of this kind. However, there is no reason to overdramatize the issues in this way.

Firstly we must remember that decisions on life and death are not (!) handed over to software systems even in self-driving cars. Algorithms are created by people. If these algorithms can learn, then they do so according to the rules laid down by the people who programmed them. The responsibility is not transferred to the system. It is passed from the individual drivers to the programmers, managers, and regulators.

Secondly these types of dilemmas rarely occur in real life on our roads, otherwise student drivers would be given training by driving instructors in how to handle them. This is obviously not happening.

Thirdly if an incident like this should occur, human drivers are not prosecuted. The way people behave in extreme situations is not classified as intentional action and the people involved cannot be held responsible or deemed to be at fault.

In the case of the software in a self-driving car, this means that it would be excessive to demand a clearly defined, ethically defensible software solution for every conceivable dilemma. We do not require this from human drivers. Instead, and this should apply to almost every area of life, we recognize that many events cannot be predicted or managed in a rational way. Tragic circumstances, where someone is in the wrong place at the wrong time, sometimes simply occur.

The role of advanced technological systems is to keep these situations to a minimum and to mitigate their consequences. It will never be possible for technology to prevent all tragedies from happening, but ethical dilemmas should not be used to discredit self-driving cars. 